\begin{tabular}{l} 
SCIENCE \& TECHNOLOGY \\
Journal homepage: http://www.pertanika.upm.edu.my/ \\
\hline PERTANIKA
\end{tabular}

\title{
SETS: A Seed-Dense-Expanding Model-Based Topological Structure for the Prediction of Overlapping Protein Complexes
}

\author{
Soheir Noori ${ }^{1,2 *}$, Nabeel Al-A'araji $\mathbf{i}^{3}$ and Eman Al-Shamery ${ }^{1}$ \\ ${ }^{\prime}$ Department of Software, University of Babylon, Babylon, Hillah, Iraq \\ ${ }^{2}$ Department of Computer Science, University of Kerbala, Karbala, Iraq \\ ${ }^{3}$ Ministry of Higher Education, Baghdad, Iraq
}

\begin{abstract}
Defining protein complexes by analysing the protein-protein interaction (PPI) networks is a crucial task in understanding the principles of a biological cell. In the last few decades, researchers have proposed numerous methods to explore the topological structure of a PPI network to detect dense protein complexes. In this paper, the overlapping protein complexes with different densities are predicted within an acceptable execution time using seed expanding model and topological structure of the PPI network (SETS). SETS depend on the relation between the seed and its neighbours. The algorithm was compared with six algorithms on six datasets: five for yeast and one for human. The results showed that SETS outperformed other algorithms in terms of F-measure, coverage rate and the number of complexes that have high similarity with real complexes.
\end{abstract}

Keywords: Common neighbours; density; protein complex; protein-protein interaction network; topological structure

ARTICLE INFO

Article history:

Received: 26 October 2020

Accepted: 18 February 2021

Published: 30 April 2021

DOI: https://doi.org/10.47836/pjst.29.2.35

E-mail addresses:

soheir.noori@uokerbala.edu.iq; soheirn.sw.hdr@student.

uobabylon.edu.iq (Soheir Noori)

nhkaghed@itnet.uobabylon.edu.iq (Nabeel Al-A'araji)

emanalshamery@itnet.uobabylon.edu.iq (Eman Al-Shamery)

* Corresponding author

\section{INTRODUCTION}

The key to exploring cell behaviour is understanding the mechanism of protein complexes. According to Pizzuti and Rombo (2014), protein complexes are a molecular aggregation of two or more proteins assembled by multiple PPIs. Protein-protein interaction (PPI) plays a central role in many biological functions and its network provides a global view of cellular functionality. 
Advanced experimental techniques have generated a vast amount of data on PPI (Wang et al., 2017). A PPI network is represented as an undirected graph, where the nodes are proteins and the edges are the interactions between proteins. Many algorithms have been proposed for the analysis of PPI networks to discover the protein complex by defining a dense subgraph in the PPI network. Two of the earliest approaches that have been adopted include the Molecular Complex Detection (MCODE) (Bader \& Hogue, 2003) and Markov (Van Dongen, 2000) algorithms. Many other algorithms search for cliques as researchers believe that a complete connected graph represents the protein complex such as Cliques Finder (CFinder) (Adamcsek et al., 2006), Clique Percolation- Distance Restriction (CPDR) (Wang et al., 2010), Maximal Cliques (Liu et al., 2009) and Local Clique Merging Algorithm (LCMA) (Li et al., 2005). All these algorithms detect cliques and then merge them depending on different criteria to identify the protein complex. Most protein complex detection algorithms such as Graph Fragmentation Algorithm (GFA) (Feng et al., 2010), Dynamic Protein Complexes (DPC) (Li et al., 2014), Detect Module from Seed Protein (DMSP) (Maraziotis et al., 2007) use the topological properties of a graph or mix the PPI network with other information like the gene expression. A subgraph is a protein complex having high functional and structural consistency (Hartwell et al., 1999). Since proteins have multiple functions, they can belong to more than one dense subgraph (Palla et al., 2005; Rives \& Galitski, 2003). Therefore, a protein complex can have an overlapping structure as is observed in the ClusterOne (Nepusz et al., 2012) and Near-Clique Mining (NCMine) (Tadaka \& Kinoshita, 2016) algorithms.

Most of the existing algorithms can detect only highly dense regions as protein complexes and ignore low density complexes (Wang et al., 2018). Further, most of them cannot detect overlapping protein complexes (Zhao \& Lei, 2019). In this study, the overlapping protein complexes with different densities are predicted through the seed expanding approach and the topological structure of the PPI networks (SETS) in an acceptable time period, namely less than five minutes for human and about one minute or less for yeast. A pre-processing step needs to be undertaken first to order the proteins according to their degrees. At the start, SETS choose the first node as a seed. Next, the direct neighbours of the seed will be added to the complex, depending on a common neighbour's technique. Notably, the proteins in a complex cannot be chosen as a next seed but can be added to another complex to generate the overlap between complexes. The predicted complex can be accepted if it is greater than the density threshold. Otherwise, all proteins of the complex will return to construct a new complex from a new seed. Finally, the preliminary complexes will be iteratively expanded according to the closeness score. This algorithm outperforms other algorithms. 


\section{METHODS}

\section{Preliminary Concepts}

Generally, PPI is represented as an undirected and unweighted graph $G=(V, E)$, where $V$ is the nodes representative of proteins and $\mathrm{E}$ is the edges which represent the interactions between the proteins. The algorithm uses several measurements.

For each vertex $v$, the degree of $v$ is the summation of its connected edges (Equation 1).

$$
d(v)=\sum_{i} e_{i}
$$

The density of the set of vertices $\mathrm{S} \subset \mathrm{V}$ is the number of edges among them divided by the number of possible edges between the set nodes (i.e., how close the set to the clique is, ranging between 0 and 1) (Equation 2).

$$
\operatorname{density}(S)=\frac{2 \times|E|}{|V| \times(|V|-1)}
$$

The common neighbours $(\mathrm{CN})$ between two proteins $\left(\mathrm{P}_{\mathrm{i}}\right.$ and $\left.\mathrm{P}_{\mathrm{j}}\right)$ are the number of proteins that indices to both divided by the square root of the product of the nodes' degrees (Equation 3).

$$
C N=\frac{N_{p_{i}} \cap N_{p_{j}}}{\sqrt{d\left(p_{i}\right) * d\left(p_{j}\right)}}
$$

\section{The Algorithm}

The results from some experiments (Goldberg \& Roth, 2003; Peng et al., 2017) have shown that the methods that used the information of common neighbours are reliable. SETS is a technique that is employed to detect overlapping protein complexes based on common neighbours. Given an undirected and non-weighted graph, the goal of the algorithm is to identify overlapping protein complexes with different densities. The algorithm accomplishes this through the following steps (Appendix 1: Algorithm 1):

1. Those proteins with a degree higher than 1 are set in ascending order, put in a queue ' $Q$ ' and its visited label is set to 'False'.

2. The preliminary complex is built starting from the seed. The nodes that share a specific ratio of common neighbours are added iteratively and its visited label is set to true if it satisfies the predefined threshold of shared neighbours in order to avoid selecting it as a seed in the next iteration. The complex will be accepted as 
a preliminary one if its density is greater than a predefined threshold. Otherwise, all the nodes visited labels will be set to 'false' and moved to the next node in the Q with a false visited label. After defining the preliminary complex, no nodes will be deleted from the $\mathrm{Q}$, so that we can get overlapping complexes. The preliminary complex will be accepted as a candidate complex if it contains more than three proteins and was not previously defined from another seed to avoid redundancy (Appendix 1: Algorithm 1, steps 1-14).

3. The candidate complex (CC) will be iteratively expanded according to the closeness score (CS) as obtained through Equation 4 by adding its neighbours ' $\mathrm{N}_{\mathrm{CC}}$ ' that connect with half or more of the proteins of the candidate complex. The expansion will be done in rounds. In each round, the algorithm will search for proteins that satisfy the threshold of closeness score $\left(\mathrm{T}_{\mathrm{CS}}\right)$ to add them to the complex. In the second round, the algorithm will search for proteins, such as those that relate to the updated complex, until no proteins can satisfy the $\mathrm{T}_{\mathrm{CS}}$. This step will assist in the identification of complexes with different densities (Appendix 1: Algorithm 1 , steps 15-20).

$$
C S(C, C u)=\frac{N_{u} \cap\left|V_{C C}\right|}{\left|V_{C C}\right|}
$$

4. Redundant complexes will be removed by retaining only one of the exactly matched complexes.

\section{Time Complexity}

The execution time has been calculated for each dataset in order to analyse the time complexity of the SETS algorithm. As a pre-processing step, SETS receive a set of ordered nodes $\mathrm{Q}$ in increasing order that take $\mathrm{O}\left(\mathrm{n}^{2}\right)$. SETS process each node in Q that has a visible label set to false and adds its neighbours. This process takes $\mathrm{O}(\mathrm{n} * \mathrm{~m})$ and reduces it to $\mathrm{O}(\mathrm{N})$ since not all nodes will be processed. Where $\mathrm{n}$ and $\mathrm{m}$ represent the number of nodes and their neighbours respectively. The second part of SETS expands each candidate complex c. This takes $\mathrm{O}(\mathrm{c} * \mathrm{~m})$, where $\mathrm{m}$ stands for the neighbours of the proteins in a complex. The time complexity of SETS is $\mathrm{O}(\mathrm{n})+\mathrm{O}\left(\mathrm{c}^{*} \mathrm{~m}\right)$. SETS is implemented in python on a 64-bit window system with a 2 GB memory and intel CPU i7 2.40 Table 1

SETS execution time in seconds GHz. Table 1 reports SETS execution time in seconds by using the time package in python.

\begin{tabular}{lc}
\hline Dataset & Time in seconds \\
\hline Collins & 0.255 \\
Gavin & 0.104 \\
Krogan & 0.191 \\
DIP & 0.893 \\
BioGRID & 67.479 \\
Human & 261.536 \\
\hline
\end{tabular}




\section{Comparison of SETS with Other Algorithms}

The performance of the algorithm has been compared to those of six others, namely MCODE (Bader \& Hogue, 2003), ClusterONE (Nepusz et al., 2012), NCMine (Tadaka \& Kinoshita, 2016), SPICi (Jiang \& Singh, 2010), IPCA (Li et al., 2008), and PEWCC (Zaki et al., 2013).

MCODE is one of the seed-extension approaches, which identifies overlapping protein complexes in three steps. Step 1, based on the core clustering coefficient, assigns a weight to every node in the graph. Step 2, extending from seeds that have a high weight, finds a dense region in the weighted graph. Finally, subgraphs that are not dense are filtered.

ClusterOne is another algorithm that detects overlapping protein complexes by starting from the seed protein having the highest degree and then gradually adding and removing proteins to find a cohesive group of proteins that can be overlapped.

On the other hand, NCMine defines a near-complete subgraph as a functional module by using the centrality degree as the weight of the nodes, which then iteratively merges these like cliques to define overlapping modules.

SPICi is a fast heuristic clustering algorithm that selects the seed having the highest weight. The weight represents the degree of the node and then uses a support function to expand the way that the density of the cluster is saved.

IPCA is another algorithm that identifies a dense region in the PPI network as a protein complex. It starts from the seed that has the biggest weight, which is the summation of its weighted edge that represents the number of its common neighbours. IPCA then recursively adds the neighbours of the seed based on two criteria: the shortest path between the seed and the node as well as the probability of its interaction.

Another algorithm that evaluates the protein interactions reliability is PEWCC. It uses the weighted clustering coefficient to detect the protein complex.

All the aforementioned algorithms rely on the topological structure of PPI networks and most of them use the seed-extension approach to detect dense protein complexes.

\section{RESULTS AND DISCUSSION}

\section{PPI and Benchmark Datasets}

The algorithm has been analysed by concentrating on five PPI networks of Saccharomyces cerevisiae (yeast) and one network of Homo sapiens (human) (Ma et al., 2017). The latter is a combination of data from two databases: HPRD (Human Protein Reference Database) and BioGRID (version 3.2.109). The PPI datasets of the yeast are Collins and Gavin for ClusterONE (Nepusz et al., 2012), DIP (Xenarios et al., 2002), Krogan (Krogan et al., 2006) and BioGRID from SPICi. Table 2 explains the properties of these datasets. Each dataset contains a different number of proteins having a different number of interactions 
that create a variety in the density of network to satisfy the diversity that is required in the PPI networks used with the algorithm. NewMIPS (Mewes et al., 2004) and CYC2008 (Pu et al., 2009) are used as benchmark complexes. All datasets are available online from authors and as Appendix 1 and 2.

Table 2

Number of proteins and intersections, and network density in PPI datasets

\begin{tabular}{cccc}
\hline Datasets & No. of Proteins & No. of Intersections & Network density \\
\hline Collins & 1622 & 9074 & 0.007 \\
Gavin & 1855 & 7669 & 0.004 \\
Krogan & 2675 & 7084 & 0.002 \\
DIP & 4930 & 17201 & 0.001 \\
BioGRID & 5361 & 85866 & 0.006 \\
Human & 15459 & 144687 & 0.001 \\
\hline
\end{tabular}

\section{Evaluation Metrics}

The quality of a predicted complex was evaluated using various metrics. The definitions of these metrics are introduced as follows:

Recall, Precision, and F-Measure. One of the metrics most commonly used to evaluate any algorithm is recall, precision and F-measure. The overlapping score (OS) is the matching score between the predicted complex $\left(\mathrm{C}_{1}\right)$ and benchmark complex $\left(\mathrm{C}_{2}\right)$, as expressed in Equation 5. $\mathrm{C}_{1}$ and $\mathrm{C}_{2}$ are considered matched if the $\mathrm{OS}$ between both is equal to or greater than 0.2 (Altaf-Ul-Amin et al., 2006; Bader \& Hogue, 2003).

$$
\operatorname{OS}\left(C_{1}, C_{2}\right)=\frac{\left|C_{1} \cap C_{2}\right|^{2}}{\left|C_{1}\right| \times\left|C_{2}\right|}
$$

Recall and precision are defined as Equation 6 and 7:

$$
\begin{aligned}
& \text { Precision }=\frac{N\left(C_{1}\right)}{\left|C_{1}\right|} \\
& \text { Recall }=\frac{N\left(C_{2}\right)}{\left|C_{2}\right|}
\end{aligned}
$$

$\mathrm{N}\left(\mathrm{C}_{1}\right)$ is the number of the predicted complex that satisfies the OS score with at least one complex in the benchmark. $\mathrm{N}\left(\mathrm{C}_{2}\right)$ is the number of the benchmark complex that satisfies the OS score with at least one predicted complex. The F-measure is a combination of recall and precision (Equation 8). 


$$
F-\text { measure }=\frac{2 \times \text { Recall } \times \text { Precision }}{\text { Recall }+ \text { Precision }}
$$

Coverage Rate (CR). CR evaluates the number of proteins that have been covered by the predicted complexes (Brohée \& van Helden, 2006; Friedel et al., 2008). CR is defined in Equation 9, where $\mathrm{C}_{2}$ is the set of benchmark complexes, maxcom $_{\mathrm{ij}}$ is the maximal common proteins between the $\mathrm{i}^{\text {th }}$ benchmark and $\mathrm{j}^{\text {th }}$ predicted complex divided by $\mathrm{N}_{\mathrm{i}}$ protein numbers in $i^{\text {th }}$ benchmark complex.

$$
C R=\frac{\sum_{i=1}^{\left|C_{2}\right|} \operatorname{Max}\left\{\operatorname{maxcom}_{i j}\right\}}{\sum_{i=1}^{\left|C_{2}\right|} N_{i}}
$$

Exact and High Matching with Real Complexes. The quality of predicted complexes was evaluated by reporting the number of real complexes that exactly match with the predicted complexes and that had an OS score greater than or equal to 0.8 , excluding the exact match.

\section{Selection of Parameters}

The $\mathrm{T}_{\mathrm{CN}}$, DT and $\mathrm{T}_{\mathrm{CS}}$ parameters had been used in SETS. Proteins that were not in the PPI network had been filtered from the benchmark complexes. Only those complexes with more than two proteins were retained and then filtered again to keep only the complexes that had all their proteins in the PPI network. CN was calculated between the proteins in the same filtered benchmark complex. We also calculated the number of complexes that at least two of their proteins satisfied the $\mathrm{CN}$ value (Appendix 2). According to the result of benchmark complexes analyses, the $\mathrm{T}_{\mathrm{CN}}$ is set for each data. Liu et al. (2010) analysed the protein complexes of CYC2008 (Pu et al., 2009), MI PS (Mewes et al., 2004) and Aloy (Aloy et al., 2004). They found that almost $60 \%$ of the complexes had a density equal to or more than 0.5 . Therefore, DT was set to 0.5 to define complexes that were dense enough to be the preliminary complexes. $\mathrm{T}_{\mathrm{CS}}$ was set to at least 0.5 to let only the proteins that had a good closeness to the preliminary complex that was to be added. Table 3 explains the threshold of each dataset.

\section{Quality of Predicted Complexes}

The performance of SETS was compared with that of six other approaches using five datasets for yeast and one dataset for human. All datasets were unweighted except SPICi, which used weighted networks. Every parameter in all the algorithms was set to default. In addition, complexes with less than three proteins were ignored. All the algorithms were implemented in the Cytoscape software (Shannon et al., 2003) except SPICi, which was 
Table 3

Threshold values for each dataset

\begin{tabular}{clll}
\hline Datasets & $\mathrm{T}_{\mathrm{CN}}$ & $\mathrm{DT}$ & $\mathrm{T}_{\mathrm{CS}}$ \\
\hline Collins & 0.3 & 0.5 & 0.6 \\
Gavin & 0.3 & 0.5 & 0.5 \\
Krogan & 0.2 & 0.5 & 0.5 \\
DIP & 0.1 & 0.5 & 0.6 \\
BioGRID & 0.2 & 0.5 & 0.7 \\
Human data & 0.1 & 0.5 & 0.7 \\
\hline
\end{tabular}

implemented in its web site. The complex is considered matched if the OS with benchmark complex is greater than or equal to 0.2. SETS have the highest F-measure in all cases and competes with other algorithms in recall and precession (Tables $4 \& 5$ ). SETS obtain the highest CR in most cases except in Collins and BioGRID, where it obtained the secondhighest CR. Besides a few exceptions where its prediction ranks behind that of the PEWCC, the exact and well-predicted complexes by SETS are the best in most cases (Figure 1). All the results are available in the Appendix 1.

The ProCope software tool (Schlicker et al., 2006) was used to evaluate the biological significance of predicted complexes and the data used in the evaluation process was set to 'default'. The evaluation was based on BP and CC. SETS detects more complexes that are

Table 4

Performance analysis for Gavin data with CYC2008 and NewMIPS

\begin{tabular}{lccccc}
\hline \multicolumn{1}{c}{ \# complex } & Recall & Precession & F-measure & CR \\
\hline Gavin with CYC2008 & & & & & \\
SPICi & 91 & 0.36 & 0.76 & 0.491 & 0.504 \\
ClusterONE & 258 & 0.508 & 0.419 & 0.459 & 0.633 \\
NCMine & 621 & 0.513 & 0.393 & 0.445 & 0.64 \\
PEWCC & 656 & 0.517 & 0.402 & 0.453 & 0.596 \\
IPCA & 464 & 0.53 & 0.457 & 0.491 & 0.626 \\
MCODE & 101 & 0.021 & 0.05 & 0.03 & 0.118 \\
SETS & 246 & 0.475 & 0.602 & $\mathbf{0 . 5 3 1}^{\text {st }}$ & $\mathbf{0 . 6 5 6}^{\text {st }}$ \\
Gavin with NewMIPS & & & & \\
SPICi & 91 & 0.372 & 0.736 & 0.494 & 0.248 \\
ClusterONE & 258 & 0.53 & 0.419 & 0.468 & 0.417 \\
NCMine & 621 & 0.549 & 0.39 & 0.456 & 0.422 \\
PEWCC & 656 & 0.552 & 0.433 & 0.485 & 0.392 \\
IPCA & 464 & 0.573 & 0.47 & 0.516 & 0.413 \\
MCODE & 101 & 0.021 & 0.059 & 0.031 & 0.045 \\
SETS & 246 & 0.524 & 0.607 & $\mathbf{0 . 5 6 3}^{\text {1st }}$ & $\mathbf{0 . 4 3}^{\text {st }}$ \\
\hline
\end{tabular}


significant in BioGRID and human datasets (Figure 2) and ranks second with regard to the rest of the datasets, competing with SPICi, IPCA and ClusterONE algorithms (Appendix 1).

SETS predict overlapping complexes as explained in Table 7 that reports some of these complexes that have high OS scores with benchmark complexes and share some of their

Table 5

Performance analysis for Krogan data with CYC2008 and NewMIPS

\begin{tabular}{lccccc}
\hline & \# complex & Recall & Precession & F-measure & CR \\
\hline Krogan with CYC2008 & & & & & \\
SPICi & 131 & 0.458 & 0.641 & 0.534 & 0.583 \\
ClusterONE & 240 & 0.492 & 0.512 & 0.502 & 0.598 \\
NCMine & 578 & 0.458 & 0.433 & 0.445 & 0.593 \\
PEWCC & 708 & 0.525 & 0.496 & 0.51 & 0.593 \\
IPCA & 472 & 0.517 & 0.595 & 0.553 & 0.599 \\
MCODE & 60 & 0.03 & 0.117 & 0.047 & 0.111 \\
SETS & 220 & 0.479 & 0.764 & $\mathbf{0 . 5 8 9}{ }^{\text {sst }}$ & $\mathbf{0 . 6 8}^{\text {st }}$ \\
Krogan with NewMIPS & & & & 0.352 \\
SPICi & 131 & 0.479 & 0.618 & 0.54 & 0.323 \\
ClusterONE & 240 & 0.442 & 0.458 & 0.45 & 0.362 \\
NCMine & 578 & 0.479 & 0.427 & 0.452 & 0.368 \\
PEWCC & 708 & 0.534 & 0.476 & 0.503 & 0.36 \\
IPCA & 472 & 0.515 & 0.574 & 0.543 & 0.038 \\
MCODE & 60 & 0.021 & 0.1 & 0.035 & $\mathbf{0 . 3 9 1}^{\text {st }}$ \\
SETS & 220 & 0.485 & 0.732 & $\mathbf{0 . 5 8 3}{ }^{\text {st }}$ & \\
\hline
\end{tabular}

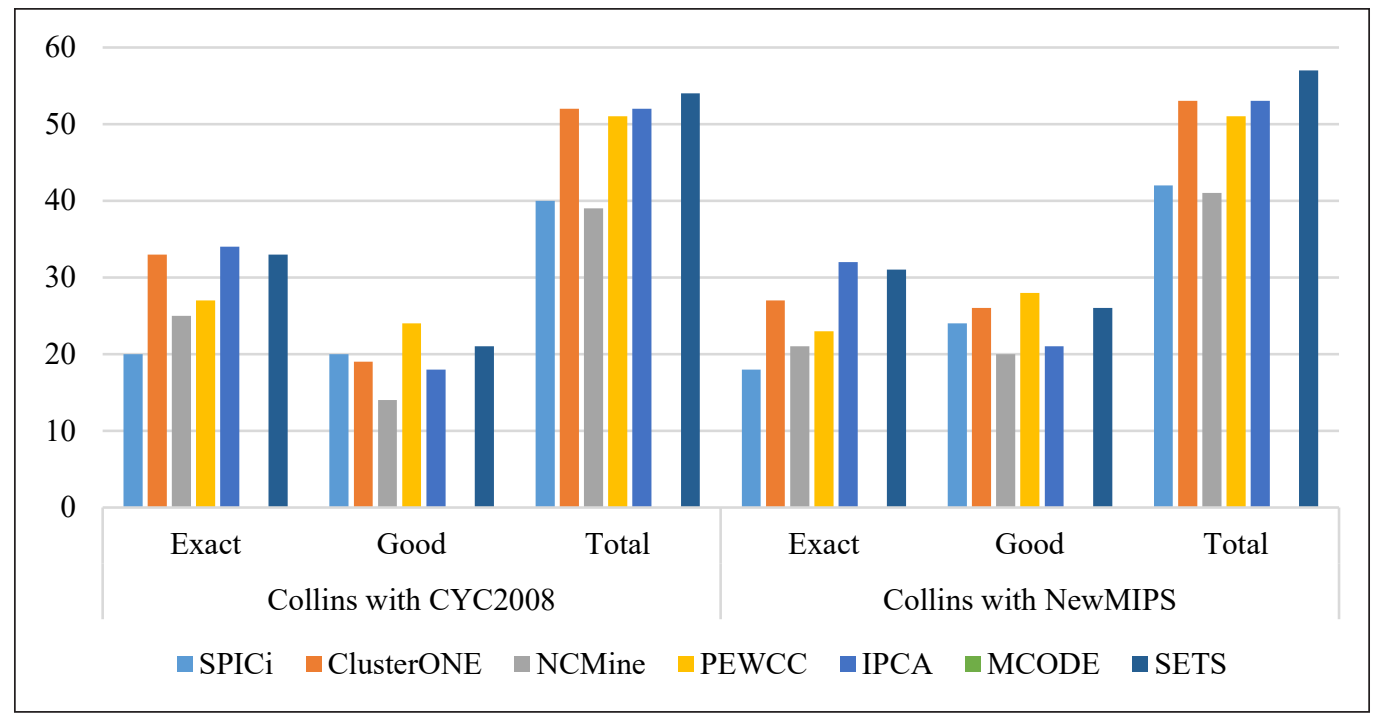

Figure 1. Number of exact and well-predicted complexes in Collins dataset 


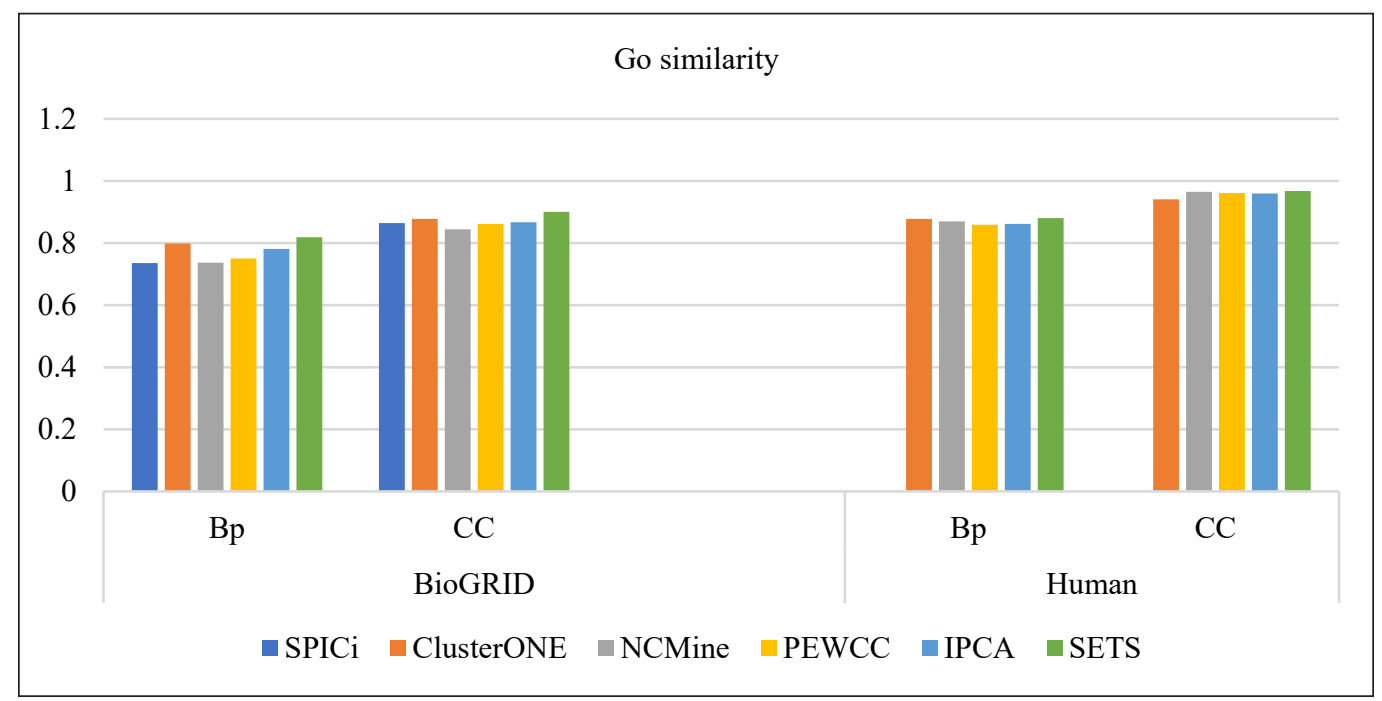

Figure 2. Biological significance of predicted complexes in BioGRID and Human

Table 6

DIP with Newmips reports low density (D.) complexes with a high OS

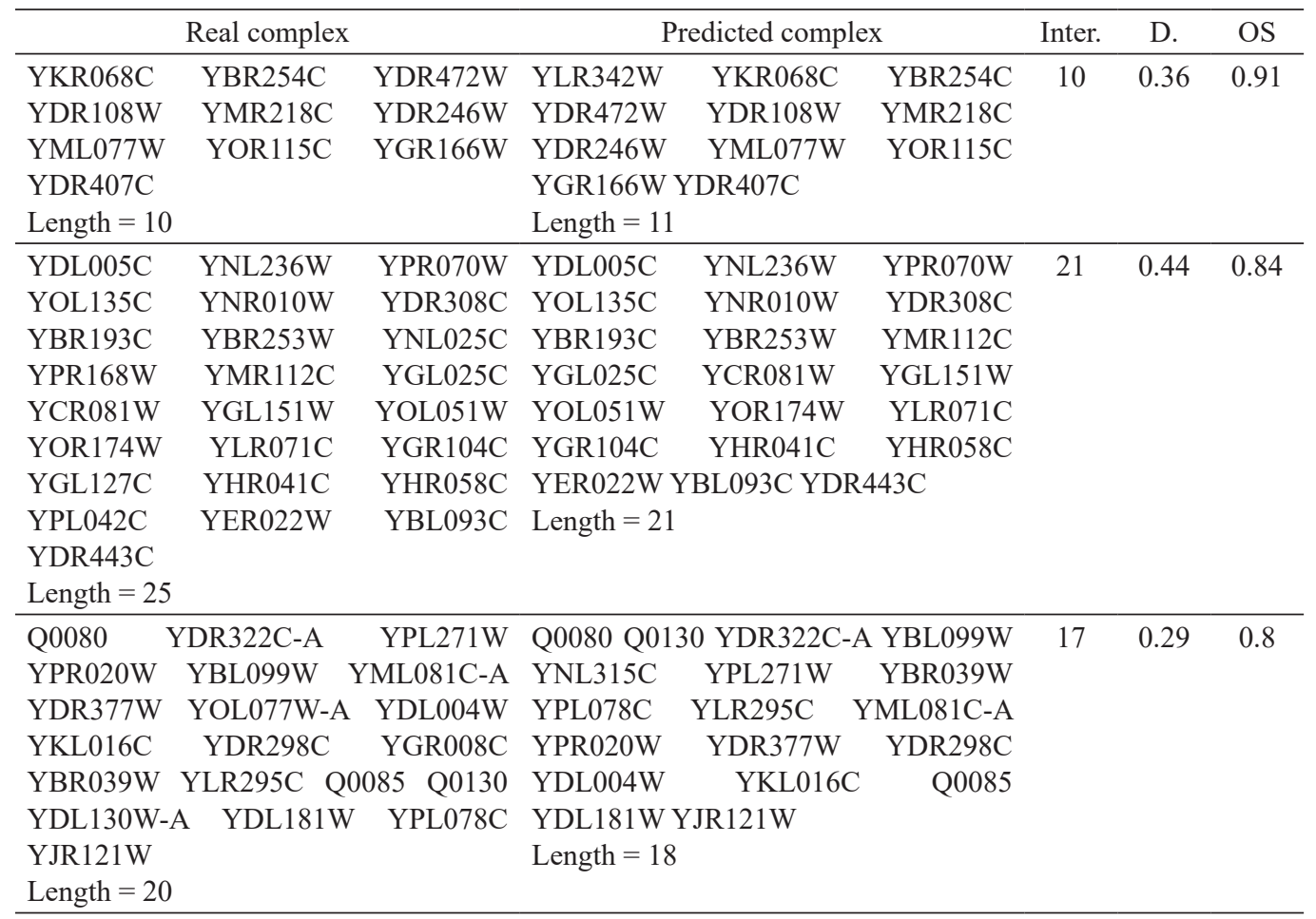


Table 6 (continue)

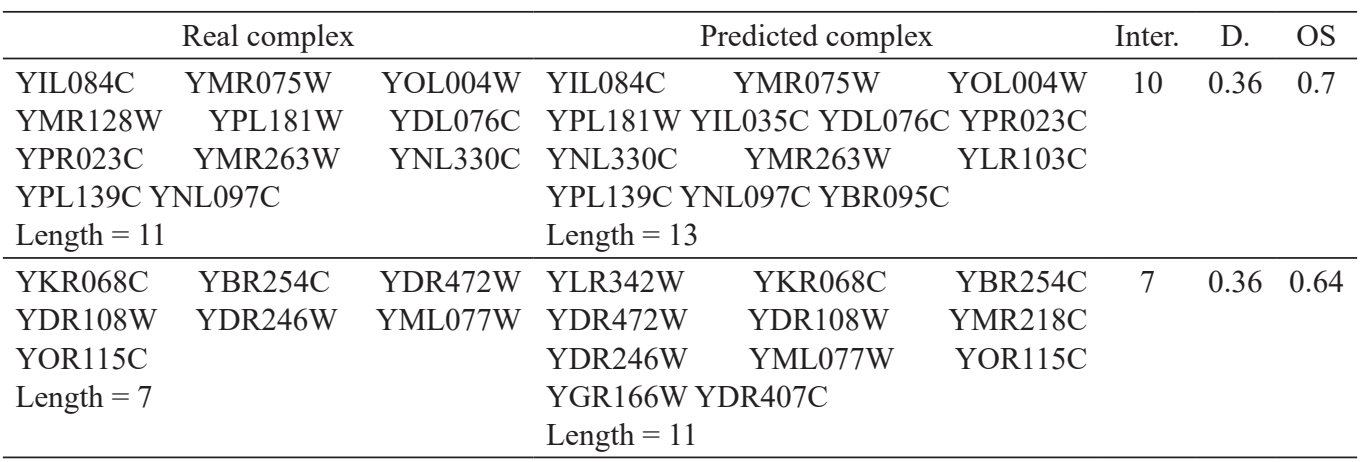

Note. Inter. is the interaction between predicted and real complexes

Table 7

Predicted overlapping complexes with high OS score from Collins using NewMIPS

\begin{tabular}{|c|c|c|c|c|c|c|}
\hline $\begin{array}{l}\text { Predicted } \\
\text { complex }\end{array}$ & Real complex & OS & $\begin{array}{l}\text { Predicted } \\
\text { complex }\end{array}$ & Real complex & OS & $\begin{array}{c}\text { Overlapping } \\
\text { proteins }\end{array}$ \\
\hline YFL039C & YFL039C & 1 & YFL039C & YFL039C & 0.92 & YNL107W \\
\hline YJL081C & YJL081C & & YDR334W & YDR334W & & YFL039C \\
\hline YOR244W & YOR244W & & YJL081C & YJL081C & & YJL081C \\
\hline YHR090C & YHR090C & & YML041C & YLR385C & & YGR002C \\
\hline YNL107W & YNL107W & & YNL107W & YML041C & & \\
\hline YNL136W & YNL136W & & YBR231C & YNL107W & & \\
\hline YEL018W & YEL018W & & YLR085C & YBR231C & & \\
\hline YHR099W & YHR099W & & YDR485C & YDR485C & & \\
\hline YPR023C & YPR023C & & YDR190C & YLR085C & & \\
\hline YFL024C & YFL024C & & YAL011W & YDR190C & & \\
\hline YJR082C & YJR082C & & YPL235W & YAL011W & & \\
\hline YDR359C & YDR359C & & YGR002C & YPL235W & & \\
\hline YGR002C & YGR002C & & & YGR002C & & \\
\hline YKL144C & YHR143W-A & 0.94 & YHR143W-A & YHR143W-A & 0.93 & YOR210W \\
\hline YOR210W & YKL144C & & YOR341W & YOR341W & & YPR110C \\
\hline YOR116C & YOR210W & & YOR210W & YOR210W & & YNL113W \\
\hline YPR190C & YOR116C & & YPR110C & YPR110C & & YBR154C \\
\hline YPR110C & YPR190C & & YNL113W & YNL113W & & YPR187W \\
\hline YNL113W & YPR110C & & YOR340C & YOR340C & & YOR224C \\
\hline YDL150W & YNL113W & & YJR063W & YJR063W & & \\
\hline YBR154C & YDL150W & & YOR151C & YBR154C & & \\
\hline YPR187W & YBR154C & & YBR154C & YNL248C & & \\
\hline YKR025W & YPR187W & & YNL248C & YDR156W & & \\
\hline YDR045C & YKR025W & & YDR156W & YPR187W & & \\
\hline YNR003C & YDR045C & & YPR187W & YPR010C & & \\
\hline YNL151C & YNR003C & & YPR010C & YOR224C & & \\
\hline YOR207C & YNL151C & & YOR224C & YJL148W & & \\
\hline YJL011C & YOR207C & & YJL148W & & & \\
\hline \multirow[t]{2}{*}{ YOR224C } & YJL011C & & & & & \\
\hline & YOR224C & & & & & \\
\hline
\end{tabular}


proteins. The complexes predicted by SETS are of various densities and not restricted to dense ones as is the case with other algorithms that use the topological structure of PPI. SETS can achieve higher F-measure with different densities of PPI network in contrast with other algorithms whose F-measure decreases when the PPI network density does. Table 6 reports some of the low-density complexes that have high OS scores with benchmark complexes.

\section{ECC vs. CN with SETS}

The CN calculation in Algorithm 1 (Appendix 1) is replaced with an edge clustering coefficient (ECC) to compare the F-measure in both cases (Figure 3). SETS with ECC is high only with Collins, which has the highest network density. It, on the other hand, achieved a lower F-measure than SETS with CN in other datasets that have different densities. Radicchi et al. (2004) realized that ECC might not suite for PPI networks as it was disassortative. This was proven with SETS that performed better using CN with different network densities.

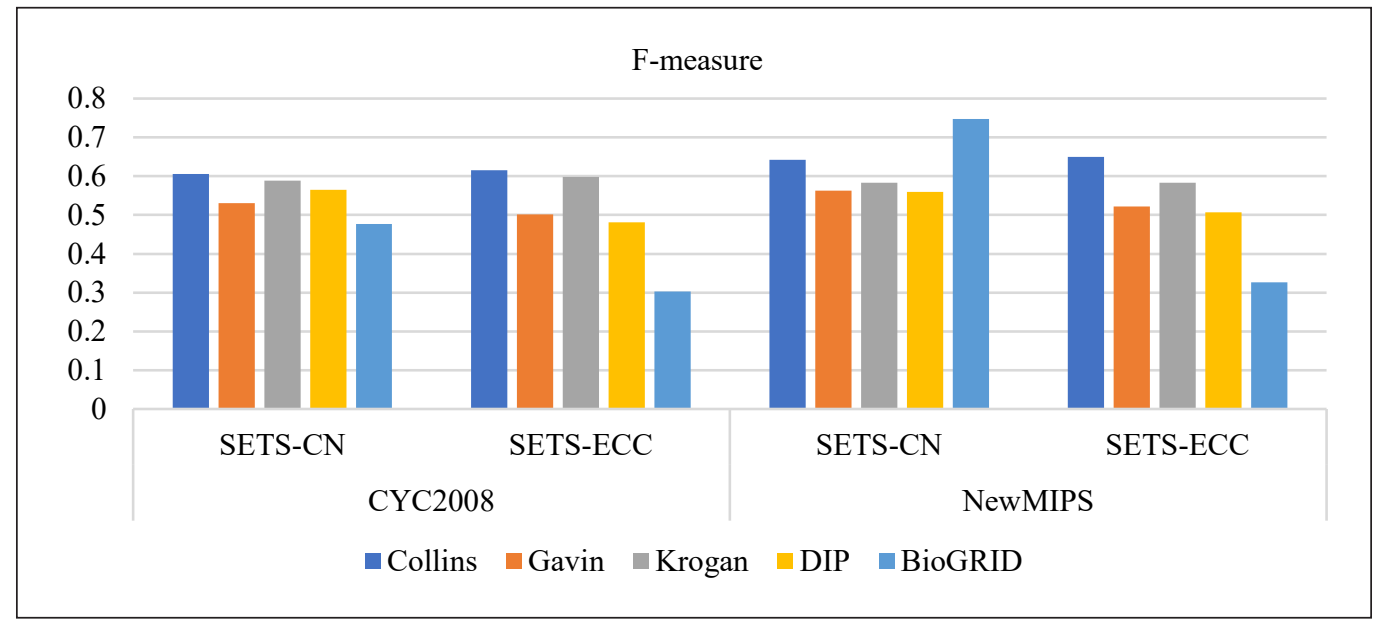

Figure 3. F-measure of ECC vs. CN with SETS

\section{CONCLUSION}

In this paper, the seed-expansion model has been proposed based on the topological structure of PPI networks to predict overlapping protein complexes with various densities. The main idea behind this algorithm is (i) choosing the first node in $\mathrm{Q}$ that is not visited before as a seed, (ii) adding the seed's neighbour that shares a specific percentage of common neighbours and accepting the complex if its density is more than or equal to the density threshold (DT) and (iii) expanding each complex by adding the proteins that are close to the complex's proteins. SETS could achieve high accuracy in all datasets that have 
different densities with good biological significance of predicted complexes compared to other methods. SETS can be further improved by using biological information as gene expression or gene ontology.

\section{ACKNOWLEDGEMENT}

We would like to thank the University of Kerbala and the University of Babylon, Iraq for partially sponsoring this research.

\section{REFERENCES}

Adamcsek, B., Palla, G., Farkas, I. J., Derényi, I., \& Vicsek, T. (2006). CFinder: locating cliques and overlapping modules in biological networks. Bioinformatics, 22(8), 1021-1023. https://doi.org/10.1093/ bioinformatics/bt1039

Aloy, P., Böttcher, B., Ceulemans, H., Leutwein, C., Mellwig, C., Fischer, S., Gavin, A.-C., Bork, P., SupertiFurga, G., \& Serrano, L. (2004). Structure-based assembly of protein complexes in yeast. Science, 303(5666), 2026-2029. https://doi.org/10.1126/science.1092645

Altaf-Ul-Amin, M., Shinbo, Y., Mihara, K., Kurokawa, K., \& Kanaya, S. (2006). Development and implementation of an algorithm for detection of protein complexes in large interaction networks. BMC Bioinformatics, 7, Article 207. https://doi.org/10.1186/1471-2105-7-207

Bader, G. D., \& Hogue, C. W. (2003). An automated method for finding molecular complexes in large protein interaction networks. BMC Bioinformatics, 4, Article 2. https://doi.org/10.1186/1471-2105-4-2

Brohée, S., \& van Helden, J. (2006). Evaluation of clustering algorithms for protein-protein interaction networks. BMC Bioinformatics, 7, Article 488. https://doi.org/10.1186/1471-2105-7-488

Feng, J., Jiang, R., \& Jiang, T. (2010). A max-flow-based approach to the identification of protein complexes using protein interaction and microarray data. IEEE/ACM Transactions on Computational Biology and Bioinformatics, 8(3), 621-634. https://doi.org/10.1109/TCBB.2010.78

Friedel, C. C., Krumsiek, J., \& Zimmer, R. (2008). Bootstrapping the interactome: unsupervised identification of protein complexes in yeast. In M. Vingron \& L. Wong (Eds.), Lecture notes in computer science: Research in computational molecular biology (Vol. 4955, pp. 3-16). Springer. https://doi.org/10.1007/9783-540-78839-3_2.

Goldberg, D. S., \& Roth, F. P. (2003). Assessing experimentally derived interactions in a small world. Proceedings of the National Academy of Sciences, USA, 100(8), 4372-4376. https://doi.org/10.1073/ pnas.0735871100

Hartwell, L. H., Hopfield, J. J., Leibler, S., \& Murray, A. W. (1999). From molecular to modular cell biology. Nature, 402(6761), C47-C52. https://doi.org/10.1038/35011540

Jiang, P., \& Singh, M. (2010). SPICi: A fast clustering algorithm for large biological networks. Bioinformatics, 26(8), 1105-1111. https://doi.org/10.1093/bioinformatics/btq078 
Krogan, N. J., Cagney, G., Yu, H., Zhong, G., Guo, X., Ignatchenko, A., Li, J., Pu, S., Datta, N., \& Tikuisis, A. P. (2006). Global landscape of protein complexes in the yeast Saccharomyces cerevisiae. Nature, 440(7084), 637-643. https://doi.org/10.1038/nature04670

Li, M., Chen, J. E., Wang, J. X., Hu, B., \& Chen, G. (2008). Modifying the DPClus algorithm for identifying protein complexes based on new topological structures. BMC Bioinformatics, 9, Article 398. https://doi. org/10.1186/1471-2105-9-398

Li, M., Chen, W., Wang, J., Wu, F. X., \& Pan, Y. (2014). Identifying dynamic protein complexes based on gene expression profiles and PPI networks. BioMed Research International, 2014, Article 375262. https://doi. org/10.1155/2014/375262

Li, X. L., Foo, C. S., Tan, S. H., \& Ng, S. K. (2005). Interaction graph mining for protein complexes using local clique merging. Genome Informatics, 16(2), 260-269. https://doi.org/10.11234/gi1990.16.2_260

Liu, G., Wong, L., \& Chua, H. N. (2009). Complex discovery from weighted PPI networks. Bioinformatics, 25(15), 1891-1897. https://doi.org/10.1093/bioinformatics/btp311

Liu, G., Yong, C. H., Wong, L., \& Chua, H. N. (2010, December 18-21 ). Decomposing PPI networks for complex discovery [Paper presentation]. 2010 IEEE International Conference on Bioinformatics and Biomedicine (BIBM), Hong Kong, China. https://doi.org/10.1109/BIBM.2010.5706577.

Ma, C. Y., Chen, Y. P. P., Berger, B., \& Liao, C. S. (2017). Identification of protein complexes by integrating multiple alignment of protein interaction networks. Bioinformatics, 33(11), 1681-1688. https://doi. org/10.1093/bioinformatics/btx043

Maraziotis, I. A., Dimitrakopoulou, K., \& Bezerianos, A. (2007). Growing functional modules from a seed protein via integration of protein interaction and gene expression data. BMC Bioinformatics, 8, Article 408. https://doi.org/10.1186/1471-2105-8-408

Mewes, H. W., Amid, C., Arnold, R., Frishman, D., Güldener, U., Mannhaupt, G., Münsterkötter, M., Pagel, P., Strack, N., \& Stümpflen, V. (2004). MIPS: Analysis and annotation of proteins from whole genomes. Nucleic Acids Research, 32(suppl_1), D41-D44. https://doi.org/10.1093/nar/gkh092

Nepusz, T., Yu, H., \& Paccanaro, A. (2012). Detecting overlapping protein complexes in protein-protein interaction networks. Nature Methods, 9(5), 471-472. https://doi.org/10.1038/nmeth.1938

Palla, G., Derényi, I., Farkas, I., \& Vicsek, T. (2005). Uncovering the overlapping community structure of complex networks in nature and society. Nature, 435(7043), 814-818. https://doi.org/10.1038/nature03607

Peng, X., Wang, J., Peng, W., Wu, F. X., \& Pan, Y. (2017). Protein-protein interactions: Detection, reliability assessment and applications. Briefings in Bioinformatics, 18(5), 798-819. https://doi.org/10.1093/bib/ bbw066

Pizzuti, C., \& Rombo, S. E. (2014). Algorithms and tools for protein-protein interaction networks clustering, with a special focus on population-based stochastic methods. Bioinformatics, 30(10), 1343-1352. https:// doi.org/10.1093/bioinformatics/btu034

Pu, S., Wong, J., Turner, B., Cho, E., \& Wodak, S. J. (2009). Up-to-date catalogues of yeast protein complexes. Nucleic Acids Research, 37(3), 825-831. https://doi.org/10.1093/nar/gkn1005 
Radicchi, F., Castellano, C., Cecconi, F., Loreto, V., \& Parisi, D. (2004). Defining and identifying communities in networks. Proceedings of the National Academy of Sciences, USA, 101(9), 2658-2663. https://doi. org/10.1073/pnas.0400054101

Rives, A. W., \& Galitski, T. (2003). Modular organization of cellular networks. Proceedings of the National Academy of Sciences, USA, 100(3), 1128-1133. https://doi.org/10.1073/pnas.0237338100

Schlicker, A., Domingues, F. S., Rahnenführer, J., \& Lengauer, T. (2006). A new measure for functional similarity of gene products based on Gene Ontology. BMC Bioinformatics, 7, Article 302. https://doi. org/10.1186/1471-2105-7-302

Shannon, P., Markiel, A., Ozier, O., Baliga, N. S., Wang, J. T., Ramage, D., Amin, N., Schwikowski, B., \& Ideker, T. (2003). Cytoscape: A software environment for integrated models of biomolecular interaction networks. Genome Research, 13(11), 2498-2504. https://doi.org/10.1101/gr.1239303

Tadaka, S., \& Kinoshita, K. (2016). NCMine: Core-peripheral based functional module detection using nearclique mining. Bioinformatics, 32(22), 3454-3460. https://doi.org/10.1093/bioinformatics/btw488

Van Dongen, S. M. (2000). Graph clustering by flow simulation [Doctoral dissertation, Utrecht University]. Utrecht University Publication. https://dspace.library.uu.nl/bitstream/handle/1874/848/full. pdf? sequence $=1 \&$ isAllowed $=\mathrm{y}$.

Wang, J., Liu, B., Li, M., \& Pan, Y. (2010). Identifying protein complexes from interaction networks based on clique percolation and distance restriction. BMC Genomics, 11, Article S10. https://doi.org/10.1186/14712164-11-S2-S10

Wang, R., Liu, G., Wang, C., Su, L., \& Sun, L. (2018). Predicting overlapping protein complexes based on core-attachment and a local modularity structure. BMC Bioinformatics, 19, Article 305. https://doi. org/10.1186/s12859-018-2309-9

Wang, Y., You, Z., Li, X., Chen, X., Jiang, T., \& Zhang, J. (2017). PCVMZM: Using the probabilistic classification vector machines model combined with a zernike moments descriptor to predict proteinprotein interactions from protein sequences. International Journal of Molecular Sciences, 18(5), Article 1029. https://doi.org/10.3390/ijms18051029

Xenarios, I., Salwinski, L., Duan, X. J., Higney, P., Kim, S. M., \& Eisenberg, D. (2002). DIP, the database of interacting proteins: A research tool for studying cellular networks of protein interactions. Nucleic Acids Research, 30(1), 303-305. https://doi.org/10.1093/nar/30.1.303

Zaki, N., Efimov, D., \& Berengueres, J. (2013). Protein complex detection using interaction reliability assessment and weighted clustering coefficient. BMC Bioinformatics, 14, Article 163. https://doi. org/10.1186/1471-2105-14-163

Zhao, J., \& Lei, X. (2019). Detecting overlapping protein complexes in weighted PPI network based on overlay network chain in quotient space. BMC Bioinformatics, 20, Article 682. https://doi.org/10.1186/ s12859-019-3256-9 


\section{APPENDIX 1}

\section{Algorithm 1}

Inputs: Q that contains ordered proteins

Output: The sets of predicted protein complexes (COMPLEXES).

1. For each protein in $\mathrm{Q}$

2. IF visited_label $==$ False

3. Add protein to complex set (COMP)

4. Set visited label of protein to True

5. For each neighbour of protein

6. Find the common neighbours $(\mathrm{CN})$ between protein and neighbours

7. IF CN $>=\mathrm{T}_{\mathrm{CN}}$

8. Add neighbour to COMP

9. Set visited_label of neighbour to True

10. IF density(COMP) $>=$ DT and COMP IS not in COMPLEXES

11. Add COMP to COMPLEXES

12. ELSE

13. For each protein in COMP

14. Set visited_label of protein to False

15. For each complex in COMPLEXES

16. For each round

17. Find neighbours $\mathrm{N}_{\mathrm{CC}}$ of complex's proteins

18. For each protein in $\mathrm{N}_{\mathrm{CC}}$

19. IF CS $(C C$, protein $)>=T_{C S}$

20. Add protein to complex

21. Return complexes

Collins

\begin{tabular}{|c|c|c|c|c|c|c|c|c|c|c|}
\hline & $\mathrm{R}$ & $\mathrm{P}$ & $\mathrm{F}$ & $\mathrm{CR}$ & $\begin{array}{c}\# \\
\text { Complexes } \\
\end{array}$ & $\begin{array}{l}\text { \# matched } \\
\text { complexes }\end{array}$ & Max & Exact & Good & Total \\
\hline \multicolumn{11}{|l|}{ CYC2008 } \\
\hline SPICi & 0.419 & 0.736 & 0.534 & 0.69 & 106 & 78 & 70 & 20 & 20 & 40 \\
\hline ClusterONE & 0.559 & 0.547 & 0.553 & 0.797 & 203 & 111 & 103 & 33 & 19 & 52 \\
\hline NCMine & 0.517 & 0.475 & 0.495 & 0.763 & 377 & 179 & 71 & 25 & 14 & 39 \\
\hline PEWCC & 0.53 & 0.521 & 0.525 & 0.738 & 426 & 222 & 89 & 27 & 24 & 51 \\
\hline IPCA & 0.542 & 0.64 & 0.587 & 0.751 & 342 & 219 & 68 & 34 & 18 & 52 \\
\hline MCODE & 0.051 & 0.107 & 0.069 & 0.121 & 103 & 11 & 79 & 0 & 0 & 0 \\
\hline SETS & 0.521 & 0.725 & 0.606 & 0.767 & 218 & 158 & 70 & 33 & 21 & 54 \\
\hline \multicolumn{11}{|l|}{ NewMips } \\
\hline SPICi & 0.473 & 0.726 & 0.573 & 0.443 & 106 & 77 & 70 & 18 & 24 & 42 \\
\hline ClusterONE & 0.588 & 0.542 & 0.564 & 0.519 & 203 & 110 & 103 & 27 & 26 & 53 \\
\hline NCMine & 0.537 & 0.501 & 0.518 & 0.493 & 377 & 189 & 71 & 21 & 20 & 41 \\
\hline PEWCC & 0.546 & 0.533 & 0.539 & 0.479 & 426 & 227 & 89 & 23 & 28 & 51 \\
\hline IPCA & 0.567 & 0.705 & 0.628 & 0.486 & 342 & 241 & 68 & 32 & 21 & 53 \\
\hline MCODE & 0.03 & 0.087 & 0.045 & 0.055 & 103 & 9 & 79 & 0 & 0 & 0 \\
\hline SETS & 0.555 & 0.761 & 0.642 & 0.488 & 218 & 166 & 70 & 31 & 26 & 57 \\
\hline
\end{tabular}

Note. R: Recall, P: Precession, F: F-measure, CR: Coverage Rate, Max: Maximum size of the complex. 


\begin{tabular}{|c|c|c|c|c|c|c|c|c|c|c|}
\hline & $\mathrm{R}$ & $\mathrm{P}$ & $\mathrm{F}$ & $\mathrm{CR}$ & $\begin{array}{c}\# \\
\text { Complexes }\end{array}$ & $\begin{array}{l}\# \text { matched } \\
\text { complexes }\end{array}$ & Max & Exact & Good & Total \\
\hline \multicolumn{11}{|l|}{ CYC2008 } \\
\hline SPICi & 0.36 & 0.76 & 0.491 & 0.504 & 91 & 70 & 13 & 14 & 10 & 24 \\
\hline ClusterONE & 0.508 & 0.419 & 0.459 & 0.633 & 258 & 108 & 40 & 11 & 22 & 33 \\
\hline NCMine & 0.513 & 0.393 & 0.445 & 0.64 & 621 & 244 & 43 & 9 & 14 & 23 \\
\hline PEWCC & 0.517 & 0.402 & 0.453 & 0.596 & 656 & 264 & 36 & 11 & 20 & 31 \\
\hline IPCA & 0.53 & 0.457 & 0.491 & 0.626 & 464 & 212 & 37 & 15 & 19 & 34 \\
\hline MCODE & 0.021 & 0.05 & 0.03 & 0.118 & 101 & 5 & 137 & 0 & 0 & 0 \\
\hline SETS & 0.475 & 0.602 & 0.531 & 0.656 & 246 & 148 & 37 & 12 & 25 & 37 \\
\hline \multicolumn{11}{|l|}{ NewMips } \\
\hline SPICi & 0.372 & 0.736 & 0.494 & 0.248 & 91 & 67 & 13 & 11 & 15 & 26 \\
\hline ClusterONE & 0.53 & 0.419 & 0.468 & 0.417 & 258 & 108 & 40 & 11 & 19 & 30 \\
\hline NCMine & 0.549 & 0.39 & 0.456 & 0.422 & 621 & 242 & 43 & 10 & 16 & 26 \\
\hline PEWCC & 0.552 & 0.433 & 0.485 & 0.392 & 656 & 284 & 36 & 13 & 21 & 34 \\
\hline IPCA & 0.573 & 0.47 & 0.516 & 0.413 & 464 & 218 & 37 & 17 & 25 & 42 \\
\hline MCODE & 0.021 & 0.059 & 0.031 & 0.045 & 101 & 6 & 137 & 0 & 0 & 0 \\
\hline SETS & 0.524 & 0.607 & 0.563 & 0.43 & 246 & 159 & 37 & 13 & 31 & 44 \\
\hline
\end{tabular}

Note. R: Recall, P: Precession, F: F-measure, CR: Coverage Rate, Max: Maximum size of the complex.

Krogan

\begin{tabular}{lcccccccccc}
\hline & $\mathrm{R}$ & $\mathrm{P}$ & $\mathrm{F}$ & $\mathrm{CR}$ & $\begin{array}{c}\# \\
\text { Complexes }\end{array}$ & $\begin{array}{c}\text { \# matched } \\
\text { complexes }\end{array}$ & Max & Exact & Good & Total \\
\hline CYC2008 & & & & & & & & & & \\
SPICi & 0.458 & 0.641 & 0.534 & 0.583 & 131 & 84 & 20 & 17 & 15 & 32 \\
ClusterONE & 0.492 & 0.512 & 0.502 & 0.598 & 240 & 123 & 23 & 12 & 15 & 27 \\
NCMine & 0.458 & 0.433 & 0.445 & 0.593 & 578 & 250 & 25 & 5 & 17 & 22 \\
PEWCC & 0.525 & 0.496 & 0.51 & 0.593 & 708 & 351 & 31 & 15 & 24 & $\mathbf{3 9}$ \\
IPCA & 0.517 & 0.595 & 0.553 & 0.599 & 472 & 281 & 22 & 19 & 15 & 34 \\
MCODE & 0.03 & 0.117 & 0.047 & 0.111 & 60 & 7 & 73 & 0 & 0 & 0 \\
SETS & 0.479 & 0.764 & $\mathbf{0 . 5 8 9}$ & $\mathbf{0 . 6 8}$ & 220 & 168 & 62 & 19 & 20 & $\mathbf{3 9}$ \\
NewMips & & & & & & & & & & \\
SPICi & 0.479 & 0.618 & 0.54 & 0.352 & 131 & 81 & 20 & 16 & 17 & 33 \\
ClusterONE & 0.442 & 0.458 & 0.45 & 0.323 & 240 & 110 & 23 & 9 & 12 & 21 \\
NCMine & 0.479 & 0.427 & 0.452 & 0.362 & 578 & 247 & 25 & 7 & 13 & 20 \\
PEWCC & 0.534 & 0.476 & 0.503 & 0.368 & 708 & 337 & 31 & 10 & 22 & 32 \\
IPCA & 0.515 & 0.574 & 0.543 & 0.36 & 472 & 271 & 22 & 13 & 18 & 31 \\
MCODE & 0.021 & 0.1 & 0.035 & 0.038 & 60 & 6 & 73 & 0 & 0 & 0 \\
SETS & 0.485 & 0.732 & $\mathbf{0 . 5 8 3}$ & $\mathbf{0 . 3 9 1}$ & 220 & 161 & 62 & 14 & 21 & $\mathbf{3 5}$ \\
\hline
\end{tabular}

Note. R: Recall, P: Precession, F: F-measure, CR: Coverage Rate, Max: Maximum size of the complex. 


\begin{tabular}{|c|c|c|c|c|c|c|c|c|c|c|}
\hline & $\mathrm{R}$ & $P$ & F & $\mathrm{CR}$ & $\begin{array}{c}\# \\
\text { Complexes }\end{array}$ & $\begin{array}{l}\text { \# matched } \\
\text { complexes }\end{array}$ & Max & Exact & Good & Total \\
\hline \multicolumn{11}{|l|}{ CYC2008 } \\
\hline SPICi & 0.555 & 0.507 & 0.53 & 0.541 & 219 & 111 & 22 & 13 & 8 & 21 \\
\hline ClusterONE & 0.436 & 0.336 & 0.38 & 0.466 & 342 & 115 & 23 & 7 & 7 & 14 \\
\hline NCMine & 0.542 & 0.291 & 0.378 & 0.497 & 1074 & 312 & 28 & 8 & 10 & 18 \\
\hline PEWCC & 0.678 & 0.317 & 0.432 & 0.582 & 1544 & 490 & 42 & 22 & 18 & 40 \\
\hline IPCA & 0.589 & 0.318 & 0.413 & 0.516 & 826 & 263 & 32 & 17 & 10 & 27 \\
\hline MCODE & 0.008 & 0.04 & 0.014 & 0.116 & 50 & 2 & 180 & 0 & 0 & 0 \\
\hline SETS & 0.653 & 0.498 & 0.565 & 0.593 & 540 & 269 & 41 & 18 & 14 & 32 \\
\hline \multicolumn{11}{|l|}{ NewMips } \\
\hline SPICi & 0.573 & 0.479 & 0.522 & 0.334 & 219 & 105 & 22 & 11 & 8 & 19 \\
\hline ClusterONE & 0.412 & 0.304 & 0.35 & 0.265 & 342 & 104 & 23 & 5 & 5 & 10 \\
\hline NCMine & 0.546 & 0.287 & 0.376 & 0.32 & 1047 & 308 & 28 & 5 & 11 & 16 \\
\hline PEWCC & 0.683 & 0.318 & 0.434 & 0.39 & 1544 & 491 & 42 & 16 & 17 & 33 \\
\hline IPCA & 0.579 & 0.311 & 0.405 & 0.323 & 826 & 257 & 32 & 18 & 8 & 26 \\
\hline MCODE & 0.006 & 0.04 & 0.011 & 0.046 & 50 & 2 & 180 & 0 & 0 & 0 \\
\hline SETS & 0.64 & 0.496 & 0.559 & 0.39 & 540 & 268 & 41 & 16 & 17 & 33 \\
\hline
\end{tabular}

Note. R: Recall, P: Precession, F: F-measure, CR: Coverage Rate, Max: Maximum size of the complex.

BioGRID

\begin{tabular}{lcccccccccc}
\hline & $\mathrm{R}$ & $\mathrm{P}$ & $\mathrm{F}$ & $\mathrm{CR}$ & $\begin{array}{c}\# \\
\text { Complexes }\end{array}$ & $\begin{array}{c}\text { \# matched } \\
\text { complexes }\end{array}$ & Max & Exact & Good & Total \\
\hline CYC2008 & & & & & & & & & & \\
SPICi & 0.432 & 0.186 & 0.26 & 0.613 & 440 & 82 & 141 & 4 & 2 & 6 \\
ClusterONE & 0.487 & 0.265 & 0.343 & 0.697 & 476 & 126 & 83 & 1 & 7 & 8 \\
NCMine & 0.737 & 0.123 & 0.211 & 0.807 & 3671 & 451 & 95 & 4 & 10 & 14 \\
PEWCC & 0.873 & 0.196 & 0.32 & $\mathbf{0 . 8 7 2}$ & 4048 & 792 & 750 & 11 & 21 & $\mathbf{3 3}$ \\
IPCA & 0.576 & 0.14 & 0.226 & 0.758 & 2718 & 381 & 80 & 2 & 14 & 16 \\
MCODE & 0.008 & 0.036 & 0.014 & 0.073 & 56 & 2 & 192 & 0 & 0 & 0 \\
SETS & 0.644 & 0.379 & $\mathbf{0 . 4 7 7}$ & 0.816 & 633 & 240 & 93 & 6 & 23 & 29 \\
NewMips & & & & & & & & & & \\
SPICi & 0.436 & 0.18 & 0.254 & 0.442 & 440 & 79 & 141 & 2 & 5 & 7 \\
ClusterONE & 0.488 & 0.25 & 0.331 & 0.496 & 476 & 119 & 83 & 1 & 6 & 7 \\
NCMine & 0.695 & 0.13 & 0.219 & 0.551 & 3671 & 478 & 95 & 5 & 8 & 13 \\
PEWCC & 0.826 & 0.21 & 0.335 & $\mathbf{0 . 6 2 7}$ & 4048 & 850 & 750 & 10 & 20 & $\mathbf{3 0}$ \\
IPCA & 0.591 & 0.138 & 0.223 & 0.538 & 2718 & 374 & 80 & 2 & 13 & 15 \\
MCODE & 0.006 & 0.036 & 0.01 & 0.029 & 56 & 2 & 192 & 0 & 0 & 0 \\
SETS & 0.622 & 0.382 & $\mathbf{0 . 4 7 4}$ & 0.561 & 633 & 242 & 93 & 6 & 21 & 27 \\
\hline
\end{tabular}

Note. R: Recall, P: Precession, F: F-measure, CR: Coverage Rate, Max: Maximum size of the complex. 
Human

\begin{tabular}{lcccccccccc}
\hline & $\mathrm{R}$ & $\mathrm{P}$ & $\mathrm{F}$ & $\mathrm{CR}$ & $\begin{array}{c}\# \\
\text { Complexes }\end{array}$ & $\begin{array}{c}\text { \# matched } \\
\text { complexes }\end{array}$ & Max & Exact & Good & Total \\
\hline SPICi & NA & NA & NA & NA & NA & NA & NA & NA & NA & NA \\
ClusterONE & 0.223 & 0.235 & 0.229 & 0.33 & 1037 & 252 & 96 & 11 & 9 & 20 \\
NCMine & 0.552 & 0.221 & 0.315 & 0.459 & 7776 & 1716 & 111 & 7 & 12 & 19 \\
PEWCC & 0.68 & 0.276 & 0.393 & $\mathbf{0 . 5 5 9}$ & 9036 & 2495 & 394 & 21 & 34 & $\mathbf{5 5}$ \\
IPCA & 0.463 & 0.266 & 0.338 & 0.455 & 6533 & 1736 & 93 & 5 & 9 & 14 \\
MCODE & 0.001 & 0.014 & 0.002 & 0.04 & 74 & 1 & 377 & 0 & 0 & 0 \\
SETS & 0.498 & 0.405 & $\mathbf{0 . 4 4 7}$ & 0.484 & 2026 & 822 & 223 & 18 & 26 & 44 \\
\hline
\end{tabular}

Note. R: Recall, P: Precession, F: F-measure, CR: Coverage Rate, Max: Maximum size of the complex.

Biological significance

\begin{tabular}{lcccccccccccc}
\hline & \multicolumn{2}{c}{ Collins } & \multicolumn{2}{c}{ Gavin } & \multicolumn{2}{c}{ Krogan } & \multicolumn{2}{c}{ DIP } & \multicolumn{2}{c}{ BioGRID } & \multicolumn{2}{c}{ Human } \\
\hline & Bp & CC & Bp & CC & Bp & CC & Bp & CC & Bp & CC & Bp & CC \\
\hline SPICi & 0.953 & 0.976 & 0.954 & 0.977 & 0.957 & 0.982 & 0.947 & 0.962 & 0.736 & 0.865 & NA & NA \\
ClusterONE & 0.902 & 0.958 & 0.782 & 0.893 & 0.849 & 0.91 & 0.809 & 0.876 & 0.799 & 0.878 & 0.879 & 0.941 \\
NCMine & 0.924 & 0.961 & 0.858 & 0.917 & 0.838 & 0.917 & 0.788 & 0.926 & 0.737 & 0.845 & 0.871 & 0.966 \\
PEWCC & 0.942 & 0.968 & 0.88 & 0.933 & 0.861 & 0.928 & 0.804 & 0.935 & 0.751 & 0.862 & 0.86 & 0.961 \\
IPCA & 0.967 & 0.981 & 0.882 & 0.937 & 0.883 & 0.938 & 0.802 & 0.946 & 0.782 & 0.868 & 0.862 & 0.96 \\
SETS & 0.962 & 0.976 & 0.897 & 0.944 & 0.897 & 0.947 & 0.833 & 0.951 & 0.82 & 0.901 & 0.881 & 0.968 \\
\hline
\end{tabular}

ECC vs. CN in SETS

\begin{tabular}{|c|c|c|c|c|c|c|}
\hline & \multicolumn{2}{|c|}{ CYC2008 } & \multicolumn{2}{|c|}{ NewMIPS } & \multicolumn{2}{|c|}{ Human - CORUN } \\
\hline & SETS-CN & SETS-ECC & SETS-CN & SETS-ECC & SETS-CN & SETS-ECC \\
\hline & F-measure & F-measure & F-measure & F-measure & F-measure & F-measure \\
\hline Collins & 0.606 & 0.615 & 0.642 & 0.65 & 0.447 & 0.333 \\
\hline Gavin & 0.53 & 0.502 & 0.563 & 0.522 & & \\
\hline Krogan & 0.589 & 0.598 & 0.583 & 0.583 & & \\
\hline DIP & 0.565 & 0.481 & 0.559 & 0.507 & & \\
\hline BioGRID & 0.477 & 0.303 & 0.474 & 0.327 & & \\
\hline
\end{tabular}




\section{APPENDIX 2}

\section{Analysis of Benchmark Complexes}

The benchmark dataset is analysed using PPI networks. Tables contain the number of proteins in each PPI as well as the number of proteins that are in benchmark complexes but are not in PPIs. The number of complexes in the benchmark dataset is reported, the benchmark complexes from proteins that are not in PPI are filtered out and only the complexes that have a length of more than two proteins are retained. The benchmark complexes are filtered again and only those complexes that have all its proteins in PPI are retained. The $\mathrm{CN}$ is calculated between the proteins of the same complex for different thresholds. The number of complexes where at least two of its proteins are satisfied at the threshold is reported and according to the number of complexes that satisfied $\mathrm{T}_{\mathrm{CN}}$ to the number of complexes from the second filter, almost $25 \%$ of complexes from the second filter, the threshold $\mathrm{T}_{\mathrm{CN}}$ is set to each PPI. The F-measure of each dataset with a different threshold proved the accuracy of the selected threshold.

Collins

\begin{tabular}{|c|c|c|c|c|c|}
\hline & $\begin{array}{l}\text { \# proteins } \\
\text { In Collins }\end{array}$ & $\begin{array}{c}\text { \# proteins in } \\
\text { benchmark but } \\
\text { not in PPI }\end{array}$ & $\begin{array}{c}\text { \# benchmark } \\
\text { complexes }\end{array}$ & First filter & Second filter \\
\hline CYC2008 & 1662 & 382 & 236 & 145 & 102 \\
\hline NewMIPS & 1662 & 695 & 328 & 221 & 106 \\
\hline $\mathrm{T}_{\mathrm{CN}}$ & CYC2008 & NewMIPS & & & \\
\hline 0.1 & 1 & 2 & & & \\
\hline 0.2 & 14 & 10 & & & \\
\hline 0.3 & 24 & 24 & & & \\
\hline 0.4 & 36 & 38 & & & \\
\hline 0.5 & 47 & 48 & & & \\
\hline $\mathrm{T}_{\mathrm{CN}}$ & \multicolumn{2}{|c|}{ F-measure (CYC2008) } & easure (NewMIPS) & & \\
\hline 0.1 & \multicolumn{2}{|c|}{0.602} & 0.638 & & \\
\hline 0.2 & \multicolumn{2}{|c|}{0.606} & 0.638 & & \\
\hline 0.3 & \multicolumn{2}{|c|}{0.606} & 0.642 & & \\
\hline 0.4 & \multicolumn{2}{|c|}{0.588} & 0.623 & & \\
\hline 0.5 & \multicolumn{2}{|c|}{0.571} & 0.604 & & \\
\hline
\end{tabular}




\begin{tabular}{|c|c|c|c|c|c|}
\hline & $\begin{array}{l}\text { \# proteins } \\
\text { In Collins }\end{array}$ & $\begin{array}{c}\text { \# proteins in } \\
\text { benchmark but } \\
\text { not in PPI }\end{array}$ & $\begin{array}{c}\text { \# benchmark } \\
\text { complexes }\end{array}$ & First filter & Second filter \\
\hline CYC2008 & 1855 & 439 & 236 & 143 & 86 \\
\hline NewMIPS & 1855 & 724 & 328 & 218 & 90 \\
\hline $\mathrm{T}_{\mathrm{CN}}$ & CYC2008 & NewMIPS & & & \\
\hline 0.1 & 5 & 6 & & & \\
\hline 0.2 & 17 & 17 & & & \\
\hline 0.3 & 25 & 29 & & & \\
\hline 0.4 & 33 & 36 & & & \\
\hline 0.5 & 47 & 51 & & & \\
\hline $\mathrm{TCN}$ & \multicolumn{2}{|c|}{ F-measure (CYC2008) } & \multicolumn{2}{|c|}{ F-measure (NewMIPS) } & \\
\hline 0.1 & \multicolumn{2}{|c|}{0.483} & \multicolumn{2}{|l|}{0.51} & \\
\hline 0.2 & \multicolumn{2}{|c|}{0.499} & \multicolumn{2}{|c|}{0.526} & \\
\hline 0.3 & \multicolumn{2}{|c|}{0.53} & \multicolumn{2}{|l|}{0.563} & \\
\hline 0.4 & \multicolumn{2}{|c|}{0.537} & \multicolumn{2}{|l|}{0.566} & \\
\hline 0.5 & \multicolumn{2}{|c|}{0.544} & 0.565 & & \\
\hline
\end{tabular}

Krogan

\begin{tabular}{|c|c|c|c|c|c|}
\hline & $\begin{array}{l}\text { \# proteins } \\
\text { In Krogan }\end{array}$ & $\begin{array}{c}\text { \# proteins in } \\
\text { benchmark but } \\
\text { not in PPI }\end{array}$ & $\begin{array}{c}\text { \# benchmark } \\
\text { complexes }\end{array}$ & First filter & Second filter \\
\hline CYC2008 & 2675 & 389 & 236 & 169 & 119 \\
\hline NewMIPS & 2675 & 604 & 328 & 249 & 123 \\
\hline $\mathrm{T}_{\mathrm{CN}}$ & CYC2008 & NewMIPS & & & \\
\hline 0.1 & 12 & 17 & & & \\
\hline 0.2 & 40 & 43 & & & \\
\hline 0.3 & 70 & 68 & & & \\
\hline 0.4 & 88 & 90 & & & \\
\hline 0.5 & 99 & 103 & & & \\
\hline $\mathrm{TCN}$ & \multicolumn{2}{|c|}{ F-measure (CYC2008) } & \multicolumn{2}{|c|}{ F-measure (NewMIPS) } & \\
\hline 0.1 & \multicolumn{2}{|r|}{0.6} & \multicolumn{2}{|c|}{0.575} & \\
\hline 0.2 & \multicolumn{2}{|r|}{0.589} & \multicolumn{2}{|c|}{0.583} & \\
\hline 0.3 & \multicolumn{2}{|r|}{0.531} & \multicolumn{2}{|c|}{0.548} & \\
\hline 0.4 & \multicolumn{2}{|r|}{0.468} & \multicolumn{2}{|c|}{0.494} & \\
\hline 0.5 & \multicolumn{2}{|r|}{0.421} & 0.442 & & \\
\hline
\end{tabular}




\begin{tabular}{|c|c|c|c|c|c|}
\hline & $\begin{array}{l}\text { \# proteins } \\
\text { In DIP }\end{array}$ & $\begin{array}{l}\text { \# proteins in } \\
\text { benchmark but } \\
\text { not in PPI }\end{array}$ & $\begin{array}{c}\text { \# benchmark } \\
\text { complexes }\end{array}$ & First filter & Second filter \\
\hline CYC2008 & 4930 & 138 & 236 & 226 & 191 \\
\hline NewMIPS & 4930 & 194 & 328 & 313 & 231 \\
\hline $\mathrm{T}_{\mathrm{CN}}$ & CYC2008 & NewMIPS & & & \\
\hline 0.1 & 61 & 100 & & & \\
\hline 0.2 & 133 & 173 & & & \\
\hline 0.3 & 158 & 201 & & & \\
\hline 0.4 & 168 & 215 & & & \\
\hline 0.5 & 170 & 218 & & & \\
\hline $\mathrm{TCN}$ & \multicolumn{2}{|c|}{ F-measure (CYC2008) } & \multicolumn{2}{|c|}{ F-measure (NewMIPS) } & \\
\hline 0.1 & \multicolumn{2}{|r|}{0.565} & 0.559 & & \\
\hline 0.2 & \multicolumn{2}{|r|}{0.516} & 0.518 & & \\
\hline 0.3 & \multicolumn{2}{|r|}{0.464} & 0.507 & & \\
\hline 0.4 & \multicolumn{2}{|r|}{0.35} & 0.407 & & \\
\hline 0.5 & \multicolumn{2}{|r|}{0.194} & 0.407 & & \\
\hline
\end{tabular}

BioGRID

\begin{tabular}{|c|c|c|c|c|c|}
\hline & $\begin{array}{l}\text { \# proteins } \\
\text { In BioGRID }\end{array}$ & $\begin{array}{c}\text { \# proteins in } \\
\text { benchmark but } \\
\text { not in PPI }\end{array}$ & $\begin{array}{c}\text { \# benchmark } \\
\text { complexes }\end{array}$ & First filter & Second filter \\
\hline CYC2008 & 5361 & 6 & 236 & 236 & 231 \\
\hline NewMIPS & 5361 & 31 & 328 & 322 & 301 \\
\hline $\mathrm{T}_{\mathrm{CN}}$ & CYC2008 & NewMIPS & & & \\
\hline 0.1 & 75 & 165 & & & \\
\hline 0.2 & 159 & 242 & & & \\
\hline 0.3 & 199 & 277 & & & \\
\hline 0.4 & 217 & 292 & & & \\
\hline 0.5 & 222 & 296 & & & \\
\hline TCN & \multicolumn{2}{|c|}{ F-measure (CYC2008) } & \multicolumn{2}{|c|}{ F-measure (NewMIPS) } & \\
\hline 0.1 & \multicolumn{2}{|r|}{0.406} & \multicolumn{2}{|c|}{0.416} & \\
\hline 0.2 & \multicolumn{2}{|r|}{0.477} & \multicolumn{2}{|c|}{0.474} & \\
\hline 0.3 & \multicolumn{2}{|r|}{0.45} & \multicolumn{2}{|c|}{0.485} & \\
\hline 0.4 & \multicolumn{2}{|r|}{0.358} & \multicolumn{2}{|c|}{0.408} & \\
\hline 0.5 & \multicolumn{2}{|r|}{0.247} & \multicolumn{2}{|c|}{0.297} & \\
\hline
\end{tabular}


SETS Algorithm for Prediction Overlapping Protein Complexes

\section{Human}

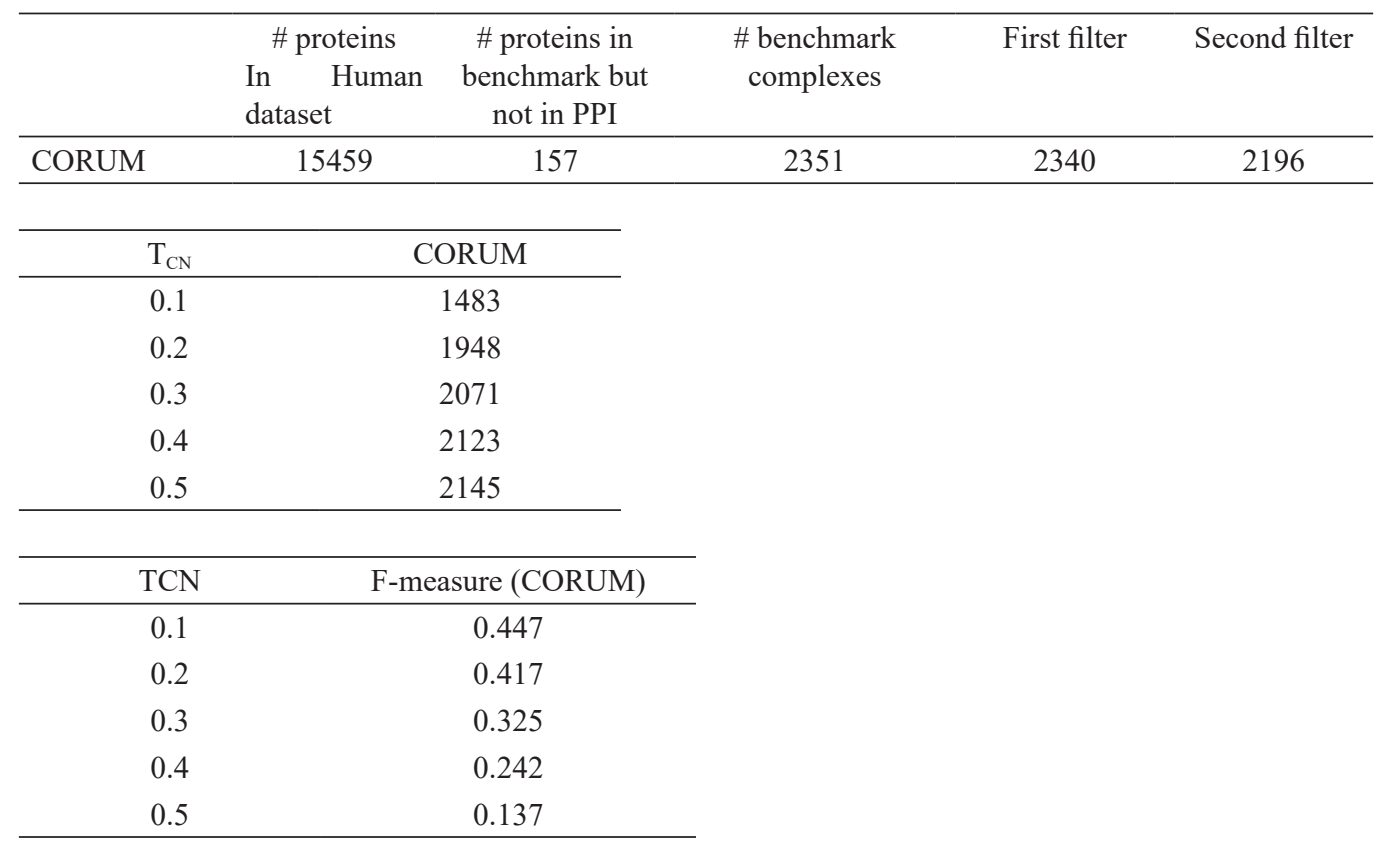


